\title{
EMERGENCY RAPID RESPONSE TO EPILEPTIC SEIZURES - A NOVEL IOT FRAMEWORK FOR SMART CITIES
}

\author{
SHABANA R. ZIYAD*AND ARMAAN ZIYAD ${ }^{\dagger}$
}

\begin{abstract}
Epilepsy is a common neurological disorder that results in seizures in patients of all ages. The frequency of seizure episodes can be controlled by prescribing anti-seizure drugs. Drug-resistant epilepsy is a condition where the seizures are uncontrolled by strong medications. Such patients are at a high risk of getting seizures frequently and prone to injuries due to sudden falls. Many countries prohibit epileptic patients from driving as sudden seizure attacks can cause loss of lives and property. In the past decades immense work has been carried out in the to monitor the seizure activity in patients and alert caregivers to extend help in emergencies. The study proposes a smart health care Internet of things framework to provide immediate help to the epileptic patient during an episode while travelling in a self-driving car. In the proposed framework, the seizure alert from a wearable device of the patient is transmitted to the control application via cloud. The control application also receives data from the vision, ultrasonic, and radar sensors. The critical information of seizure alert and the sensor data commands the car to force stop. The seizure google map location of the car is sent to the patient's caregiver as well as the registered hospital. Many applications are being developed to provide luxury and comfort to fully automated car drivers. Far from providing luxury to the driver Emergency Rapid Response to Epileptic Seizure aims to propose a solution that could save the life of an epileptic patient who is drug-resistant or prone to frequent attacks despite severe medications. The experimental results on synchonization of clouds show that the minimum time is $30 \mathrm{sec} 30 \mathrm{~ms}$ and maximum time is $31 \mathrm{sec} 63 \mathrm{~ms}$. The experimental results prove that its recommended to alert the patient's caregiver directly from control application rather than alerting via cloud.
\end{abstract}

Key words: Internet of Things, Automobile Sensors, Self-driving cars, Wearable devices, Epilepsy, Seizures

AMS subject classifications. $68 \mathrm{M} 14$

1. Introduction. Epilepsy is a set of chronic neurological disorders caused by abnormal discharge in brain neurons, which is characterized by unprovoked and re-current seizures [1]. Around 50 million people in the world suffer from epilepsy ${ }^{1}$. With timely diagnosis and effective treatment, epileptic patients can be seizure-free after a couple of years of medication. In most low-income countries, the lack of effective diagnosis and non-availability of anti-epileptic drugs has led to patients suffering from seizures all their life. Despite of prolonged medications patients suffer from sudden and unpredictable seizure attacks. The unpredictable nature of seizure attacks develops anxiety and stress for the patient and their family. The epileptic patients are forced to be accompanied by caregivers. Epileptic seizures can be classified as Tonic-clonic (TCS), Myoclonic, Clonic, Tonic, and Atonic. Tonic-clonic is a condition where the patients lose consciousness followed by violent jerking and body stiffness. Myoclonic involves jerking which resembles jerking due to electric shocks. Clonic is repetitive, rhythmic jerking movements. Tonic is a seizure that causes muscle stiffness and rigidity. Atonic is a seizure that causes a general loss of muscle tone ${ }^{2}$.

Most epileptic seizure patients are on medications that reduce the number of episodes during their lifetime. Some epileptic seizures cannot be controlled by drugs and are referred to as Drug-Resistant Epilepsy (DRE). Such patients may have to undergo surgeries as part of treatment ${ }^{3}$. The patients whether on medication or drug-resistant are at higher risk of getting seizures when they are under stress. Driving is a daily activity that makes a person independent to do their tasks. The task of driving can be dangerous for epileptic patients when a seizure attack occurs while driving. The accidents caused due to seizures can lead to vehicle crashes and deaths. The risk increases while driving vehicles whether it is automated cars or partially automated cars.

* This work was supported by the Deanship of Scientific Research, Prince Sattam bin Abdulaziz University. Department of Computer Science, College of Computer Engineering and Sciences, Prince Sattam Bin Abdulaziz University, Al Kharj, Kingdom of Saudi Arabia (ziyadshabana@gmail.com)

$\dagger$ CS Academy, Kovaipudur, Coimbatore, India 
This results in damage to public or private property and a threat to public safety [2]. Epileptic patients are prohibited from driving in certain countries but developing countries do not have such restrictions.

The recent technological developments in automotive engineering, have led to the development of innovative and safe cars. Artificial intelligence algorithms which are efficient in predicting and forecasting issues have found application in automotive engineering. In the past two decades, the automotive industry has witnessed remarkable development in the field of automation of vehicles. There is a substantial increase in investment in the design of semi -automated and automated cars. Smart vehicles are the future of smart cities. The manufacturers now integrate the concepts of Artificial intelligence, including Machine learning and deep learning with automotive technology to design smart cars. The manufactures now incorporate the features of driver assists, connected vehicles, smart parking, lane switching based on sensors in the cars. The automobile sensors acquire data from the surroundings and are processed by the inbuilt control application in the car. This system has full control over the car to act without human intervention. In this study, we propose an Internet of Things (IoT) framework for smart health care in smart cities.

The advancement in cloud computing technologies integrated with IOT has witnessed the development of several health care applications in smart cities. Smart health care is found to provide a timely and effective response to patients in an emergency condition. Apart from data collection, analysis, and monitoring new health care applications emerge every day to provide emergency care. The epileptic patient having a seizure is found unconscious and compulsorily need immediate help. The patient needs some first aid like turning them to one side to allow them to breathe freely and loosen their clothes to make them feel comfortable. In case of any injuries, the patient needs immediate medical attention. In most cases due to organ contractions that occur in seizures the patients are at risk of vomiting. Hence immediate attention is required in such cases. An unattended patient recovers consciousness, but the patient suffers from severe fatigue and confusion. Due to this health condition, they are unable to call for any help. These risk factors are of prime concern for the families of epileptic patients who are unable to drive by themselves. The patients become dependent on others for their daily tasks of travelling. There is a pressing need for a health care solution that would make the dream of several epileptic patients come true. The health care solution empowers the patients to drive by themselves and provides emergency help in case of need. The health care monitoring and response framework proposed in this study is a milestone in health care research that ensures patient safety while driving.

This research paper is the first of its kind that focuses on a proposed model which provides emergency help for patients during the onset of seizures while driving cars. This proposed model of IoT, Emergency Rapid Response to Epileptic Seizures (ERRE) will prove to be an effective system to help seizure patients from meeting with accidents in the event of getting a seizure attack in a self-driving vehicle. It also alerts the patient's family and nearby hospital about the patient's status and location. The IOT model includes wearable devices to detect seizures which alerts the car's inbuilt control application. The control application based on the data from automobile sensors stops the car seconds within alerting the vehicles nearby. The car sensors detect the vehicles and objects moving near the patient's car and alerts the cars to break in the case of an emergency. The implementation and performance evaluation of the framework is the future direction of work. Section 2 is the related literature study of the applications and frameworks for epileptic patient monitoring system. Section 3 throws light on the background of the paper. Section 4 explains the architecture of the proposed model for Emergency Rapid Response to Epileptic Seizure. The section 5 is the result and discussions sections. Section 6 is the conclusion and future direction of the study. The Figure 1.1 shows the block diagram of the proposed system ERRE.

2. Related Study. Epilepsy patients require immediate attention on getting seizures as they are prone to injuries due to losing consciousness suddenly [3]. There are several applications developed for seizure management all over the world. One such application is Epi \& me which provides efficient management of seizures in children and adults by communicating and storing data of seizures and treatment. It records the events of seizures, their occurrence, detects factors that trigger seizures, creates awareness of the side effects of medications, assists in treatment, and tracks the movement while the patient is asleep. It also provides a graphical

\footnotetext{
${ }^{1}$ https://www.who.int/health-topics/epilepsy

${ }^{2}$ https://my.clevelandclinic.org/health/diseases/9917-epilepsy-types-and-their-symptoms

${ }^{3}$ https://epilepsysociety.org.uk/about-epilepsy/drug-resistant-epilepsy
} 


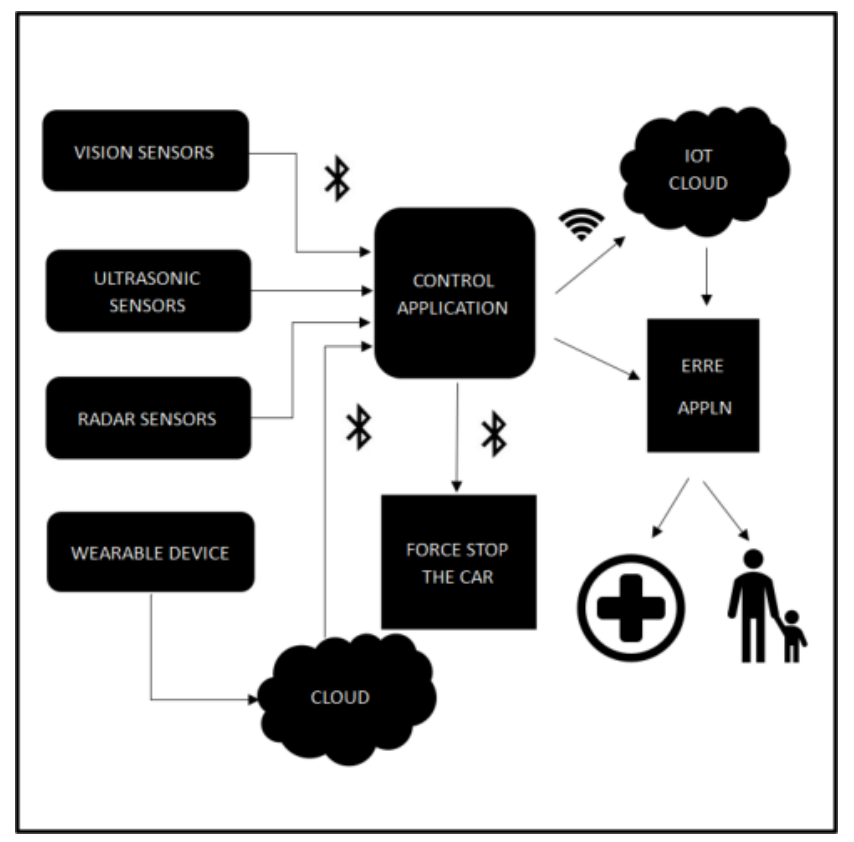

FIG. 1.1. Block Diagram for Proposed ERRE

representation of the frequency of occurrence of seizure attacks and video recordings of such episodes. A smart app called Epilepsy Self Monitor EpSMon, has emerged as a collaborative effort of clinicians, the National Health Service (NHS), and epilepsy charity's Sudden Unexpected Death in Epilepsy (SUDEP) Action. This app monitors the health condition of the patients based on a set of questionnaires and suggests the patients have more regular medical reviews. This in turn results in seizure control and accidents due to seizures ${ }^{4}$. Eppdetect, is an application that identifies a potential seizure by sensing movements that last for more than a period of 10 seconds ${ }^{5}$. The seizure tracker is an app that allows the parents of children suffering from seizures to record the details of each episode of the seizure. Epilepsy Foundation of Minnesota has developed an application called Seizure First Aide which provides first aid instructions to the person who attends to an epileptic patient. This lists out the steps to follow in case of a seizure attack in the patient ${ }^{6}$. ICE Medical Standard has created a facility that allows the patient to share information such as contact numbers, medication details to the first responder on the patient's phone's lock screen. This ensures rapid response in case of emergency. Epilepsy Health Storylines is an app designed to be more than just a seizure tracker. The app provides a variety of tools that manages a patient's condition. Its comprehensive feature set includes recording symptoms, seizures, moods, and setting reminders for taking medication. Epilepsy Health Storylines is designed to provide a variety of tools to manage patients. Its features include recording symptoms, the occurrence of seizures, setting reminders for medication according to prescriptions, and detect mood patterns ${ }^{7}$. SeizAlarm is an app that monitors repetitive motion and detects increased heart rate to alert the contact person to alarm the occurrence of seizure. This feature can be disabled while playing or engaging in any physical activity which results in increased heart rate to avoid false alarm ${ }^{8}$.

In some apps, location is sent to the contact person to locate the patient. The system proposed by authors [4] is a cloud-based IoT framework that includes EEG sensors to record and transmit EEG signals of the patients. The framework decides to send the alert to the deep learning module based on the patient's real-time

\footnotetext{
${ }^{4}$ https://sudep.org/epilepsy-self-monitor

${ }^{5}$ https://www.mdedge.com/neurology/epilepsyresourcecenter/article/103200/epilepsy-seizures/smartphone-apps-help-manage

${ }^{6}$ https://www.medicalnewstoday.com/articles/319430\#ICE-Medical-Standard

${ }^{7}$ https://healthstorylines.com

${ }^{8}$ http://seizalarm.com/about/
} 
data. The features for epilepsy detection include the patient's body movements, gestures, actions, and facial expressions to classify the seizure condition. The data from EEG sensors as well as the features perform seizure detection in the cloud. On detecting seizure conditions the alert is given to medical practitioners. The model achieves accuracy and sensitivity of 99.2 and 93.5 percent, respectively.

The architecture proposed in research work by [5] is a solution for epilepsy monitoring. The system includes a wearable device and a smartphone which are portable and is near the patient all the time. The two wearable devices are linked by Bluetooth 4.0 to a Smartphone. This is addressed as a patient kit. The types of epilepsy on which this system focuses are tonic-clonic seizures and absence seizures. This type of epileptic seizure can be detected by triaxial accelerometer and heart rate sensors. The battery life can be extended by a suitable balancing algorithm. A study is carried out by researchers to maintain a balance by decreasing the communication activity and decreasing the computation amount. In addition to ontology-driven tasks, the system also performs dynamic data gathering personalization. An Adhoc decentralized solution Mobile Ad Hoc Networks is implemented. MANNET demands heavy and computationally expensive cloud services. The smartphone and the app together with the ontology perform the sensor sampling. The data transfer takes place with Wi-Fi. Connectivity facilitates notifications and alarms. In addition, specific hardware performs the function of a federated Cloud computing server. MANET Mobicloud, has the potential to support distributed and collaborative CC services. The main tasks include receiving and storing data from wearable devices. The second task performs partition the data and in turn sending requests to CC or MCC.

This research has achieved its goal of detecting seizures, receiving and maintain data from sensors, and alerting the medical staff. This drawback of this research work is that it focuses only on a specific type of seizure called tonic-clonic seizures. In the IoT framework proposed by [6] the EEG recording is measured and communicated via Bluetooth to FGPA which has an embedded deep learning algorithm. The EEG data along with prediction results is sent to Rasberry pi. The classification results in fire an alarm to the patient's caregiver. A Deep Convolution neural network extracts the features of the brain during the preictal state. The IoT network also stores the EEG data in the cloud to be referred by doctors. The Raspberry Pi 3+ device with WiFi sends the data to a cloud-based platform. The performance analysis of the classification techniques such as SVM, CNN, and DCNN are recorded. The DCNN shows improved results compared to other classification models. It gives an accuracy of 96.1 percent, sensitivity of 97.41 percent, and specificity of 94.8 percent. The security and privacy issues of the wearable devices, WiFi connectivity vulnerability are the drawbacks of this system. In the IoT-based epilepsy monitoring system proposed by [7] the data from sensors for measuring temperature, accelerometer, sound are retrieved. The fall in temperature, convulsions of limbs, and sound due to fall are measured. The microcontroller and the RF modem which is a handset to manage RF correspondence and transmit baud rates from CMOS/TTL source. The data is stored in the database for future reference.

This research work does address the security issues and does not alarm the patient relatives regarding the occurrence of seizures. The IoT framework proposed by [8] acquired EEG data and it is pre-processed and communicated through gateways to the Cloud. The preprocessing of data is followed by feature extraction before performing classification. The proposed system classifies the data and allows health care professionals to access the data to provide emergency care. The patient data is stored in the cloud and can be accessed by IoT devices. This study is a proposed model and focuses on the classification of the data. An EEG-based epileptic seizure detection is developed for the cognitive health care framework. EEG signals from the scalp of the patients, psychological signals, movements, facial expressions, and gestures of the patient are collected and communicated to the cognitive system. Facial expressions from health care are transmitted to the cognitive system and the system makes decisions regarding the detection of seizures. An alert is given to the patient's caregiver. In the cloud, seizure detection is performed. The results of seizure detection are sent to the health care professionals and other stakeholders.

This paper proposes a novel integration of deep CNN and stacked autoencoders. Deep CNN is adopted due to the artifacts in EEG signals and hurdles in feature extractions [9]. There are many frameworks developed for monitoring epileptic patients and providing smart health care by leveraging the facilities of IoT. The research work proposed in this paper is the first of its kind to provide emergency care to a patient with a sudden epileptic seizure attack who is driving in a self-driving car. The framework commands the control application to force stop the car based on the data received from wearable devices and car sensors. The EEG data is preprocessed 
by DWT and the feature extraction is performed. The statistical features are extracted from the raw data. Naïve Bayes classifier classifies the presence of seizures. The system is validated with Simulink, ThingSpeak, and available microcontrollers. Experimental results record a classification accuracy of 98.65 percent [10].

3. Background. This section throws light on the ERRE framework concepts. This section discusses the different types of wearable devices that can detect the onset of epileptic seizure attacks. IOT automobile is a technology that makes the driving experience easier and safer for drivers. This section summarizes the distinct features of automated self-driving cars. The significance, working, and architecture of IoT are also discussed in this section.

3.1. Wearable Devices. Wearable devices and their application in health care. Wearable devices are pragmatic in continuous patient monitoring, detection, diagnosis, and therapy. Smartwatches and wrist bands are popular wearable devices among health care wearable devices. These devices are designed to monitor the health parameters which in turn reduces the risk due to lifestyle-related diseases. The compatibility of wearable devices with other devices like smartphones and applications has led to reliable health care monitoring systems. Availability of wearable devices for measuring health parameters and bio-signals claims to detect several disease conditions including epilepsy. The range of bio-signals and routine scalp electroencephalogram (EEG) was monitored by wearable devices. Real-time data acquisition of the patients by Empatica's E4 wrist band and Everion upper armband proves effective in monitoring the patient's condition. Sensors include surface electromyography (sEMG) wires and Epilog scalp patch electrodes. Devices that are wearable and removable proves to be fruitful in monitoring the health condition of patients [11]. Seizure detection wearable devices can be classified into four types of models based on the biomarker used for epilepsy detection.

3.1.1. Conventional Wearable EEG Devices. The wearable EEG devices are available as the headband and headset-type devices. These devices are efficient in measuring the EEG signals from the forehead over the prefrontal cortex. It measures factors such as attention, emotion, and cognition [12,13]. The headset-type EEG devices can measure EEG signals from brain areas, including the parietal, temporal, and occipital cortices. The EEG acquiring wearable devices employ their electrode configurations. The disadvantage in this device is that the electrodes should be in tight contact with the scalp surface in the hair-bearing area [14]. EPOC+ by Emotiv (phase 1 study) has an accurate recall of 39 percent with a precision of 71 percent and an F1-score of $0.51[15]$.

3.1.2. Wearable device based on accelerometry-based sensors. The devices which are designed on accelerometry sensors are Smartwatches. Smart Monitor (recall of 31percent) is provided with an alarm facility that alerts the caregivers. Wii Remote has a recall of 100 percent and an F1-score of 0.86. The i -pad Touch has a recall of 87 percent and an F1-score of 0.40 . The epi alert has a recall of 91percent and F1-score of 0.80 and Epicare has a recall of around 90 percent. [16].

3.1.3. Wearable device based on Electromyography. Electromyography detects muscle contractions during an epileptic seizure. EDDI (phase 3 study) has an inbuilt sEMG sensor which is worn on the upper arm with a recall of 94 percent in the detection of Tonic-clonic seizure (TCS) [17]. SPEAC, the Brain Sentinel Monitor and Alerting System, showed a recall of 95 percent with an F1-score of 0.95 and a low FAR of 0.017 in the detection of TCS [18].

3.1.4. Hybrid Models. The Nightwatch measures ACM and PPG with an accuracy of 86 percent and an F1-score of 0.62. It ensured rapid response to caregivers [19]. The Empatica wristband has sensors for Accelerometry (ACM), Electrodermal Activity (EDA), temperature, and photoplethysmography (PPG). The recall rate is between 92 and 100 percent [20]. Embrace is a wristband that has EDA and ACM sensors for the detection of TCS. The recall rate is 94 percent and low precision of 10 percent with an F1-score of 0.18 [21]. The hybrid models are preferable compared to the other models in efficient detection of seizure in TCS. Figure 3.1 represents the working of the Embrace Smart monitor for epileptic seizures.

3.2. Automated Self-driving Cars. In a smart city, the recent technological developments have led to the new trend of automated cars. The environmental data collected from the surrounding area of the cars using sensors are processed by the inbuilt-CPU of the car effectively to provide partial or full automation. The data 
collected from sensors like radar, pressure, temperature, image detection, oxygen sensors, ultrasonic sensors, promotive, rain, humidity, speed, parking sensors aid the CPU on board to make the accurate decision and enhance the driving comfort. The rapid advances in the field of microelectronic devices are a milestone in the development of automated cars. Automated cars integrate the high-tech features of robotic systems. In case of emergency, automated cars have lesser responses time to act compared to human-driven vehicles [22].

3.2.1. Tesla Autopilot. Tesla one of the leading automobile manufacturers presents autopilot cars with full driving capability. Tesla delivers that "Autopilot is intended for use with a fully attentive driver, who has their hands on the wheel and is prepared to take over at any moment" 9 . Tesla cars are provided with onboard computers with cameras and sensors which enable a smooth self-driving experience for the drivers. Full driving capability includes features like an auto lane change, auto park, and smart summon. Safety features available in these cars are automatic emergency braking, front collision warning, side collision warning, obstacle aware acceleration, blind-spot monitoring, lane departure avoidance. The cruise control is a feature that allows the speed of Tesla cars to be adjusted based on the position of the car ahead. This is enabled by radar and forwardfacing cameras. If any vehicle is detected in the lane in front of the car the speed of the car is automatically adjusted. The autosteer function ensures that the Tesla cars stay centered in a lane as well as assist in change lanes, and self-park. To ensure that Tesla cars are centered, the road markings are detected by the cameras and the sensors monitor the vehicles available on the road to maintain a safe distance. The sensors ensure that the cars do not collide with other cars. The cameras and sensors assist in the self-park of the cars without colliding with sides ${ }^{10}$. Level 2 automated system has full control of the vehicle but in case of emergency, the driver must be ready to intervene. Tesla Autopilot is an Advanced Driver-Assistance System feature that provides autonomous driving. This technology provides the capacity to automatically shift lanes and summon cars to and from the parking lot. Autopilot includes track-aware cruise control and autosteer facilities. Autopilot is loaded with hardware that adopts deep learning techniques to adapt to real-time situations ${ }^{11}$. The built-in controller identifies the location of the car in its reference map and updates the control instructions according to events in the surroundings of the car. The control application relies on the sensor to change the position of the car. The Tesla cars are the future which has advanced features of automation which include track-aware cruise control, lane centering, self-parking, automatic lane changes, summon a car from the parking lot, and navigation.

3.3. Internet of Things. Internet of things includes sensors, actuators, processors, software systems, humans, and communication channels to enable effective communication between entities. This powerful concept has found its application in all fields including industries, health care, transport, smart homes, and smart cities. The technological growth in IoT has paved the way for new innovative technological solutions to Heath care issues. The novel technological framework developed in IoT has aided in monitoring the patient status more effectively. Each IoT develops an independent network of intelligent objects that can communicate with each other. Each IoT implements a unique service and new communication methods between things. Internet of things is not an independent technology it is an aggregation or cluster of distinct technologies that collaborate and serve to make life easier for users. IoT applications are the future that will provide solutions to various diversified problems. IoT addresses issues of heterogeneous device management, communication, integration, and security. Figure 3.1 showcases a graphical representation of IoT. Data synchronization is the process of synchronizing the data between two or more clouds such that any updates or changes in data are automatically reflected. This ensures consistency within the systems. Data synchronization is growing in importance as there is an escalated accessibility to cloud-based data in IoT. In health care, the consistency of data is an important factor as there is frequent updating of data. The issues of consistency and security are also to be addressed. Healthcare compliance is a very critical issue and can be catastrophic in case of failure. Time is the prime factor in healthcare, and to provide efficient patient care there is a demand for collaboration tools to keep pace with time. There is no compromise on security and scalability with data moving to the cloud [23].

\footnotetext{
${ }^{9}$ https://www.tesla.com/support/autopilot. Accessed August 12, 2020.

${ }^{10}$ https://www.jameco.com/Jameco/workshop/Howitworks/how-it-works-tesla-autopilot-self-driving-automobiletechnology.html

${ }^{11}$ https://www.tesla.com/support/autopilot
} 


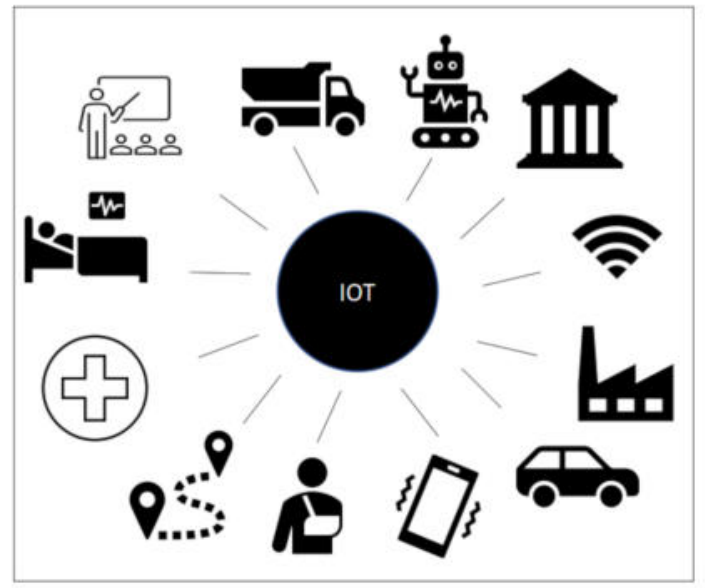

FIG. 3.1. Internet of Things

3.4. Architecture for IOT. The two main IoT architecture for designing solutions in IoT include Reference Architecture and Internet of things architecture. Reference Architecture is applicable for Industries IoT and Internet of things architecture is intended for any IoT solution. These architectures are popularly used for any reliable structure. The architectures of IoT are broadly classified as three-layer and five-layer architecture.

The three-layer architecture includes Application, Network, and Perception layers. The five-layer architecture includes the Business, Application, Processing, Transport, and Perception layer. The business layer manages the IoT system including the applications and business models. The application layer delivers the specific service to users which could be a service related to smart cities, smart homes, smart factories, or smart health. The processing layer includes the databases and data processing capabilities that process the data from lower layers to higher layers. The transport layer is dedicated to transfer sensor data from the lower layer of the architecture to the processing layer through the communication protocols. The Physical layer integrates all the information from smart objects necessary for the smart application [24]. The figure 3.2 represents the five layers of IOT architecture.

4. Architecture of Proposed Framework - EERE. The Emergency Rapid Response to Epileptic Seizure is an IOT that provides rapid action to epileptic patients in automated self-driving cars. The fivelayer the architecture of the proposed model includes the Perception, Transport, Processing, Application, and Business layers. The perception layer is the lowest layer of the architecture that collects data from sensors. The sensors used in this proposed model includes the wearable device to alert epilepsy, visual sensors, radar sensors, ultrasonic sensors of the automated self-driving cars.

4.1. Perception Layer of ERRE. The perception layer is the physical layer which is a collection of sensors, network devices, and cloud servers. The perception layer of EERE includes the wearable devices that alert seizure episodes, sensors of the automated cars, the cloud servers.

4.1.1. Epilepsy Seizure Detection Sensors. The wearable devices for the detection of epilepsy proved to be a boon to patients who are drug-resistant and are suffering from tonic-clonic seizures. They are lifesaving devices that alert the family or friends regarding the seizure attack thereby avoiding sudden death due to seizures. The physiological condition of the patient is the biomarker to detect an epileptic seizure. One such biomarker is electrodermal activity. Electrodermal activity measures the skin conductance which is affected by the sweating of the skin. Sweating arises due to emotional and psychological stress in epileptic patients during a seizure attack. The skin conductance value increases with the sweat gland activity. EDA is measured in units called micro siemens. The EDA has two electrodes, and a small alternating current is passed to the skin through the electrodes. The measurement data vary between the left and right wrist. The hydration level in a 


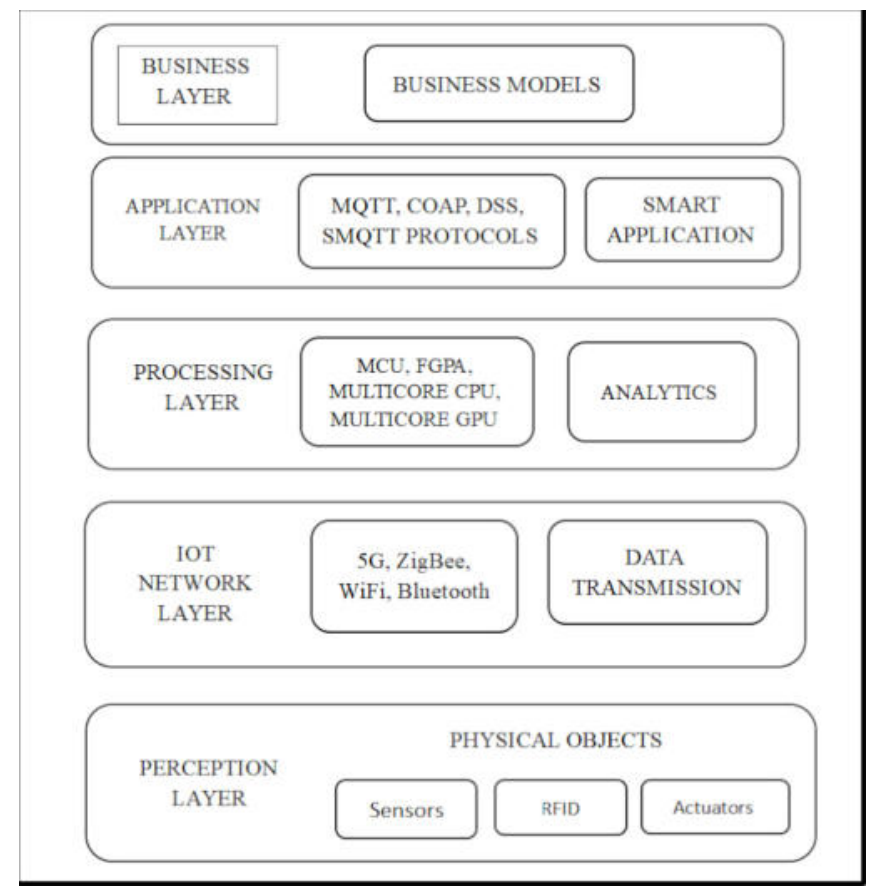

FIG. 3.2. Five Layers Architecture of Internet of Things

person's body also affects the EDA measurements. The temperature of the patient is measured by an optical infrared thermometer located in the EDA. This technology provides faster measurement without contact with skin. The optical sensor measures the temperature accurately. Epileptic seizures cause body temperature to fall considerably compared to the regular body temperature. This reduction in temperature is a biomarker for generalized tonic-clonic seizures (GTCS) detection. The tri-axial accelerometer (3DACC) is a sensor that measures the rhythmic movement of the GTCS occurring in patients. The 3DACC generates the data related to raw acceleration, body acceleration, and gravity. Its default value is $-2 \mathrm{~g}$ to $+2 \mathrm{~g}$.

The accelerometer measures linear motion, whereas the gyroscope sensor is used to measure the orientation and angular velocity of the patient. It measures the motion of the patient [25]. The double T-structure made up of crystal material is an internal vibrating element. This T-structure holds a stationary part in the center to which a sensing arm is attached. The drive arm found on both sides is symmetrical in structure. A continuous lateral vibration is generated due to the alternating vibration of the electrical field. The leaking vibrations are handled by the left and right movement of drive arms. The signal resolution is in 8 bits. When a GTCS is detected, the wearable device alerts the patient's family through the Alert app. Embrace2 is a wrist-worn biosensor device that detects the seizure by sensors. Embrace 2 has an inbuilt CPU, memory chip, Bluetooth antenna, and LEDs along with sensors. Embrace2 measures the electrodermal activity, motion activity, and skin temperature of epileptic patients. These physiological parameters are communicated to the Alert and Mate app. These data are then transferred to Empatica's cloud. The Embrace biosensor device has sensors of Electrodermal activity, temperature, Accelerometer, and Gyroscope . A machine learning algorithm with the data from the sensors predicts the onset of seizures in patients. The Mate app records the physical activity, details of seizure episodes, and sends alerts to the Patient's caregiver. The Figure 4.1 shows the working of Empatica, Seizure detection device ${ }^{12}$.

4.1.2. Automobile Sensors. Automobile sensors are intelligent sensors that control and process levels of coolant and oil. Some car sensors include mass airflow sensor, engine speed sensor, oxygen sensor, voltage sensor, and vehicle speed sensor. In addition to these, there are sensors for object tracking and detection in

\footnotetext{
12 www.empatica.com/en-eu/embrace2/
} 


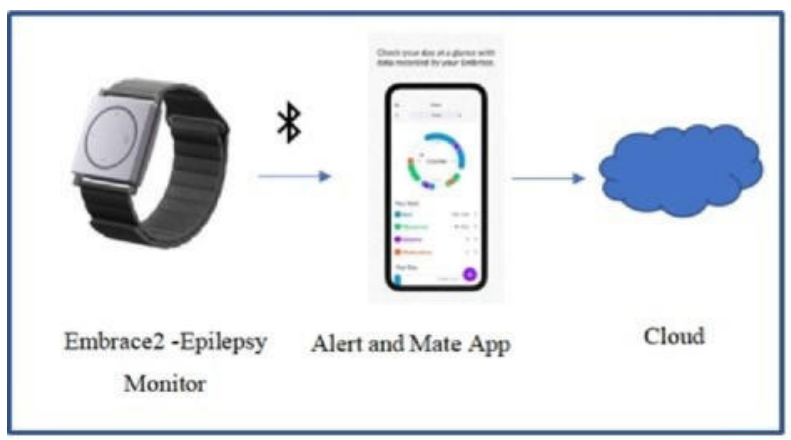

FIG. 4.1. Empatica Wearable Device for Epilepsy Detection

automated cars. These sensors collect data from the surroundings of the vehicle and transfer the data to the control application for further processing.

Self-driving cars can drive and maintain the car at a safe distance from other cars. It can automatically break when there is a car or pedestrian in the moving direction of the car. This technology is implemented by motion detection and object tracking. This detection and tracking devices retrieve data from the camera sensors which detects and alerts the driver if the car is on autopilot mode. These sensors could be used for the ERRE framework as the power consumption is less in these sensors. In addition to power saving, they also provide efficient and flexible data transfer to the processors. This makes these types of sensors more suitable for image processing in embedded IoT. The photons that fall on the photodiodes are converted and stored in capacitors. The voltage of the capacitor is converted into digital signals by analog signal chain and analog to digital converter. The vision sensor, CMOS image sensors converts the charge accumulated to a voltage at the pixel level. The image processor performs the processing of the image and transfers it to the main processor which is FPGA. Ultrasonic sensors emit ultrasonic waves of sound and convert the sound that has been converted to an electrical signal to detect the distance between the car and the objects. One of the sensors to detect an object is the ultrasonic sensor which assists in automatic parking. These sensors measure the proximity of the object by calculating the propagation time. The sensor has a piezoelectric transducer, printed circuit board, and microcontroller for signal transfer. The piezoelectric transducer converts electric charges to vibrations and vice versa. Applying a voltage to the electrodes generates the acoustic waves. Acoustic waves can generate voltages in the device. The frequency band is between 40 to $50 \mathrm{kHz}$. The electronic control unit in the sensor causes the circuit to excite the Piezoelectric transducer, which causes the membrane attached to vibrate and emit pings. Ultrasonic sensors cannot detect objects which are in very close proximity as they cannot receive signals before the ring downtime which is approximately $700 \mu \mathrm{s}$ [27]. The ultrasonic sensors detect objects around the car and that information helps in automatically switching lanes.

Radar sensors detect objects around them by analyzing the signals that are electromagnetic waves transmitted to extract the information of objects. Radar uses the doppler property of electromagnetic waves to detect the speed and position of the objects. The Doppler effect is the wave frequency shift that is between the source and destination. The time delay and phase shift assist in the measurement of the range and Doppler velocity of the objects. Radars are available at different frequencies varying from $24 \mathrm{GHz}$ to $79 \mathrm{GHz}$. The recent technology uses $79 \mathrm{GHz}$ for automotive radars. The advantage of using a radar sensor is that it works well in all climatic conditions, but it falsely detects road signs, rails, and stationary objects. Unlike the camera sensors, the radar works well even in bad weather conditions such as fog, dust, smoke, and rain [28]. For automated cars, short-range sensors allow blind-spot monitoring and parking assistance whereas long-range radar sensors assist in automatic distance control. The radar sensors of the car can view up to $160 \mathrm{~m}$ ahead through sand, snow, and fog along with the cameras. The automated cars use short-range radar for cruise control, collision proximity warning, and blind-spot detection.

4.2. IOT Gateway Layer of ERRE. The gateway layer of ERRE performs a translation of protocols between the perception layer with sensors and the internet layer. The gateways act as a bridge between the 
Internet layer and the sensor network. Edge computing facilitates a geo-distributed network of smart gateways between sensors and the cloud layer. This provides advantages of load balancing, mobility, and scalability. The wearable devices do not facilitate storing patient data. One approach is to transfer the data to the cloud and store them in the cloud. An effective solution is fog computing. The context data from sensors or smart wearable devices are transferred to the fog layer and then to the cloud. The fog or edge layer is the intermediate layer that provides the extended cloud computing service for IoT. The data from the wearable device to detect GTCS is now transferred to the fog layer by Bluetooth technology. Protocol conversion is performed on data from different subnetworks. The backend is the cloud that performs the storing and computation.

4.3. Processing Layer of ERRE. The selection of sensors and processors for IoT device is an important factor that affects the efficiency of IoT design. The available processors are General Purpose Processors (GPP), Digital Signal Processors (DSP), Microcontroller Units (MCU), Graphics Processing Units (GPU), Field Programmable Gate Arrays (FPGA), and System-On-a-Chip (SOC) devices. The processor for the IoT must be chosen carefully. The GPP is designed for computers and the compatibility with IoT devices is limited. It requires additional peripherals to carry out IOT functions which prove to be expensive. Microcontrollers are standalone devices suitable for image processing in IoT. Microcontrollers do not possess a Very Long Instruction Word (VLIW) architecture; hence the parallel execution of multiple instructions is challenging. DSPs adopt the VLIW architecture. Multiple instructions are executed simultaneously implementing the feature of parallelism, which is an advantage in image processing. DSP is more suitable for computing simple programs, but memory is a constraint. Logic programming is supported by FPGA. The FPGA architecture has multiple logic gates compared to the DSP. FPGA allows parallelism which makes it a suitable IoT processor. FPGA is a better power-draining component compared to other processors. Inexpensive FPGA chips with low power are developed for IoT applications [29]. In this proposed design of IoT FPGA will be the suitable processor.

4.4. Application Layer of ERRE. Protocols are essential to eliminate the problem of interoperability between the application and the internet. The application of ERRE and the protocol are part of the application layer of the IoT which delivers application-oriented services. Some of the protocols for the Application layer of IoT include Message Queue Telemetry Transport (MQTT), Constrained Application Protocol (CoAP), Data Distribution Service (DDS), Secure Message Queue Telemetry Transport (SMQTT), and Representational State Transfer (RESTFUL SERVICES). In this ERRE we propose SMQTT which is apt for sensor networks. SMQTT is a lightweight protocol based on the publish-subscribe architecture model. EERE falls under the publishsubscribe architecture. The patient's epileptic condition is published to the server. The subscribed clients and in this case receive information through the broker. The data from the sensors are published by the broker, an IoT device. This data is published with a key. The client in this case may be a tablet or mobile. These clients receive sensor data by establishing a connection with the broker. The data from the sensor is sent to the requesting client through the broker. In exceptional situations of a connection failure due to low bandwidth, the protocol manages the crucial situation. The publisher sends encrypted data which is decrypted by the subscriber. The sensor which detects GTCS publishes the data and the caregiver's smart device will decrypt the data. This protocol is adopted to provide secure data transfer [30]. Hence the critical issue of security in data transfer in IoT is also addressed in this framework of EERE.

5. High Level Architecture of ERRE. The epileptic seizure alert from the wearable device is transmitted to the cloud. On receiving an alert, the control application requests and receives the automobile sensor data. The data from the vision sensor, ultrasonic sensors, and radar sensors detect the proximity to the patient's car. On detecting that the other cars are at safe distance from the patient's car, the patient's car is commanded to force stop. The control application now sends the current location of the car to the cloud. The IoT cloud sends the location to the smart device to the patient's caregiver. The framework ERRE gives an alert to the registered hospital. Retrieve the data from a wearable device for seizure alert. If an Epilepsy seizure is detected, then retrieve the data from car sensors. The radar, camera, and ultrasonic sensors detect objects by sensor fusion technology. The control application gives the command to stop the car. The data is transmitted to the IoT cloud. The control application also transmits the message to the patient's family informing the onset of the seizure The ERRE App sends the current location of the car to the caregiver and registered hospital. The patient data is stored in the database in the cloud for future use by the medical team. 


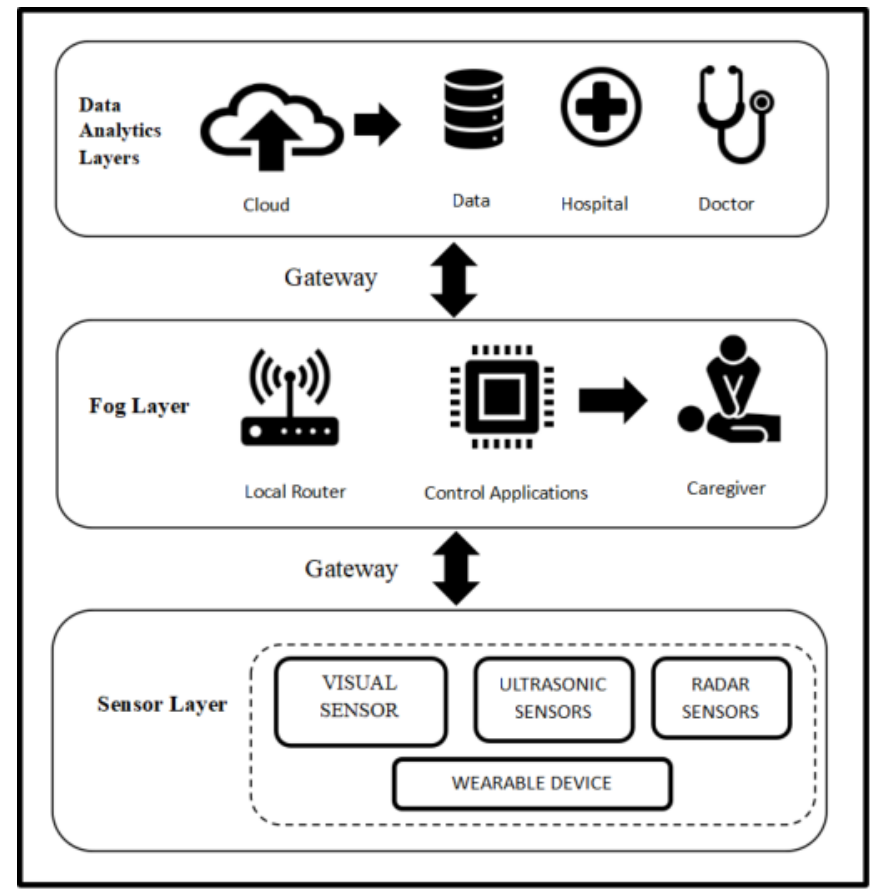

FIG. 5.1. High Level Architecture of ERRE

Step 1: Retrieve the data from wearable device for seizure alert.

Step 2: If Epilepsy seizure detected, then retrieve the data from car sensors.

Step 3: The radar, camera and ultrasonic sensors detect objects by sensor fusion technology.

Step 4: The control application gives command to stop the car.

Step 5: The data is transmitted to the IOT cloud.

Step 6: The control application also transmits the message to patient's family informing the onset of seizure Step 7: The ERRE App sends the current location of the car to the family member and registered hospital. Step 8: The patient data is stored in the database in the cloud for future use by medical team.

The high-level architecture has three layers namely the Sensor layer, Fog layer, and Cloud layer [31]. The sensor layer performs sensor fusion and low-level data fusion. Sensor calibration and sensor fusion are important concepts in automated vehicles. Sensor calibration explores the actual position of the object in the surrounding. The exact calibration of the sensor is the prerequisite for sensor fusion. Sensor fusion allows data from multiple sensors to be fused to reduce the contradictions. Camera-Radar fusion (CR fusion) is a unique technology that integrates data from camera and radar for object detection. The low-level fusion approach allows each sensor like the radar, ultrasonic, and camera to carry out the object detection and tracking independently. The data collected from the seizure detection sensors and the car sensors are relatively small and the fusion is performed on board. This fusion of streaming sensor data and seizure alert sensor data facilitates emergency alert. The middle layer is the fog layer which has the data onlooker nodes and n-fog nodes [32]. The data on-looker nodes perform data filtering and filter redundant data and generate the seizure alarm. The fog nodes are responsible for classifying the user's current health status based on the fused sensor data. The fused sensor data is combined with the static information of the patient and is transferred to the higher layer. This is the middle-level data fusion. The high-level layer is the IOT cloud layer that maintains the data of the patient for future reference by medical practitioners. The high-level data fusion enables the fusion of the patient data with the existing database in the cloud [33]. On detecting a seizure alert from the wearable device, the control application based on the data from the sensors force stops the car. The car location is sent to the patient's relatives to trace the car. The High level Architecture of ERRE is showcased in Figure 5.1. 


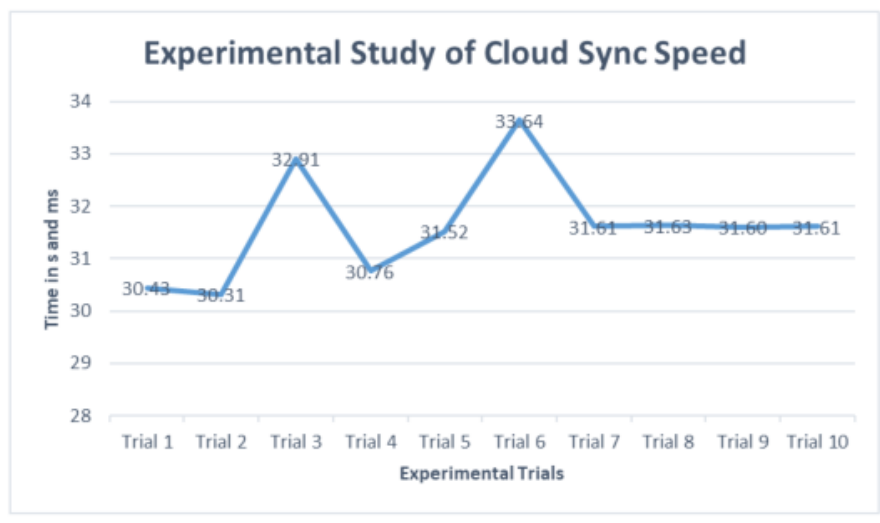

FiG. 6.1. Experimental Results of the Cloud Synchronization

6. Result and Discussion. The experiment study includes evaluating the transfer speed and the cloud syncing speed. The cloud chosen for this experiment is google drive. We have synced and transferred data across clouds and measured the transfer rate. The syncing of data is an essential component for an IoT framework. The syncing is performed by a professional data synchronization system. To evaluate the performance of the part of the IoT framework a syncing of the drives was performed. The trial runs of the data synchronization are performed, and the results are showcased as a graph in Figure 6.1. The experimental results show that the synchronization of clouds shows minimum time is $30 \mathrm{~s} 30 \mathrm{~ms}$, maximum time is $31 \mathrm{sec} 63 \mathrm{~ms}$. Processing the data in the cloud layer increases the latency of emergency events. It is not appropriate to handle large-scale systems and it is unsuitable for mobile users. Hence the emergency alert will be directly sent to the person concerned from the control application of the car. The control application of the automated cars possesses a built-in feature to send the location of the car to external entities. On receiving an emergency alert the patient can receive help within seconds which can be lifesaving for an unattended epileptic patient. The map coordinates of the car with GPS location of the car are sent to the patient's caregiver. This enables the patient's relative to use google maps and trace the location of the car. The message delivered includes a text message "HELP!" along with coordinates. Code is executed in Python language.

7. Conclusion. The revolutionary changes in technology in the fields of automobiles and networking have led to the emergence of several IoT-based smart health care solutions. Health care is one such field that demands continuous synchronization of the data in the cloud with scalability at a low cost. Several cloud providers support data storage and sharing among the users. This facilitates the patient to stay connected to the family and health care practitioners. Several applications are available for patient status monitoring and alerting the caregivers in case of emergency. This alerts the medical team to recah the patient in emergency condition. Smart health care solution allows the continuous monitoring of the patient's health condition through IoT. The ERRE is one a novel IOT framework which alerts the patient's family on a seizure episode while driving a selfdriving car. The proposed IOT framework is an integration of sensors that include vision sensor, radar sensor, ultrasonic sensor to detect the object near the car.The wearable device has sensors that can alert the onset of the seizure. The control application on receiving the alert from the wearable device sends the command to stops the car and alerts the patient's caregiver and the registered hospital. The patient's family can trace the car's location and immediate medical help will be provided within a short time. This prevents the epileptic patient in the car to remain unattended due to seizures. Among the IoT framework for epileptic seizure monitoring, this proposed framework will be beneficial for epileptic patients who are drug-resistant or suffer from frequent seizure episodes. ERRE is the first of its kind to provide emergency response for the epileptic patient in the self-driving car. To the best of knowledge this is a unique work which proves to be lifesaving for an unattended epileptic patient. There are many IOT framework available which automate the tasks of users to provide luxury a nd comfort to them. This framework provides emergency help to the patient in critical condition. The future research work includes the implementation of the complete IoT framework. The IoT framework performance evaluation is also part of future research work. Such health applications create confidence in the patients to live independently as they are constantly monitored by caregivers and the medical team. 
Acknowledgement. I would like to express our deep gratitude to Dr. Haya Alaskar, Vice Dean, College of Computer Engineering and Sciences (Female Section), Prince Sattam Bin Abdulaziz University, Al Kharj, KSA, for her constant support and valuable guidance in the research work. This publication was supported by the Deanship of Scientific Research at Prince Sattam Bin Abdulaziz University, Al Kharj, Saudi Arabia.

\section{REFERENCES}

[1] F. Mormann and R. G. Andrzejak, Seizure prediction: Making mileage on the long and winding road, Brain, 139(6), (2016), pp. 1625-1627.

[2] A.Krumholz, R.S. Fisher, R.P. Lesser, W.A. Hauser, Driving and epilepsy: A review and reappraisal, JAMA, 256, (1991),pp. 622-626.

[3] H.Blumenfeld , Impaired consciousness in epilepsy, Lancet Neurol , 11(9), (2012), pp.814-826.

[4] M. Alhussein, G. Muhammad, M. Shamim Hossain, S.U. Amin , Cognitive IoT-Cloud Integration for Smart Healthcare: Case Study for Epileptic Seizure Detection and Monitoring, Mobile Networks and Applications, 23, (2018), pp. 1624-1635.

[5] P.M. Vergara, Enrique de la Cal, José R. Villar, Víctor M. González, Javier Sedano, An IoT Platform for Epilepsy Monitoring and Supervising, Journal of Sensors, 2017, (2017), pp. 6043069

[6] H. Daoud, P. Williams and M. BayoumiIo T based Efficient Epileptic Seizure Prediction System Using Deep Learning", IEEE 6th World Forum on Internet of Things, (2020), pp. 1-6

[7] P.T.Jagtap, N.P Bhosale, IOT Based Epilepsy Monitoring using Accelerometer sensor, International Conference on Information , Communication, Engineering and Technology, (2018), pp. 1-3

[8] A.K. Gupta, C.Chakraborty, B. Gupta, Monitoring of Epileptical Patients Using Cloud-Enabled Health-IoT System ,Traitement du Signal, 36(5), (2019), pp. 425-431

[9] Alhussein, M., Muhammad, G., Hossain et al, Cognitive IoT-Cloud Integration for Smart Healthcare: Case Study for Epileptic Seizure Detection and Monitoring, Mobile Netw Appl, (2018), 23, pp. 1624-163

[10] A. Sayeed, S. P. Mohanty, E. Kougianos, V. P. Yanambaka and H. ZaveriA Robust and Fast Seizure Detector for IoT Edge, Proceedings of IEEE International Symposium on Smart Electronic Systems, (2018), pp. 156-160

[11] E. Bruno, A. Biondi, S. Böttcher, S. Lees, A.S. Bonhage, M.P. Richardson, Day and night comfort and stability on the body of four wearable devices for seizure detection: A direct user-experience, Epilepsy \& Behav, 112, (2020), pp. 107478.

[12] M. Beauregard, J. Courtemanche, V. Paquette , Brain activity in near-death experiencers during a meditative state, Resuscitation, 80, (2009), pp. 1006-1010.

[13] N.H LiU, C.Y. ChiAng, H.C CHU, Recognizing the degree of human attention using EEG signals from mobile sensors, Sensors, 13, (2013), pp. 10273-10286.

[14] S. PARK; C.H. HAN, , C.H. IM, Design of Wearable EEG Devices Specialized for Passive Brain-Computer Interface Applications, Sensors, 20, (2020), pp. 4572-85.

[15] Mckenzie ED, Lim ASP, Leung ECW, Cole AJ, Lam AD, Eloyan A ET AL, Validation of a smartphone-based EEG among people with epilepsy: a prospective study, Sci Rep,7, (2017), pp. 1-8.

[16] J. Verdru, W. VAn Paesschen, Wearable seizure detection devices in refractory epilepsy, Acta Neurol Belg,120, (2020), pp. $1271-1281$.

[17] S. Beniczky S, I.Conradsen ,O. Henning ,M. Fabricius , P.Wolf , Automated real-time detection of tonic-clonic seizures using a wearable EMG device, Neurology, 90(5), (2018), pp. e428-e434.

[18] C.Á. Szabõ, L.C. Morgan , K.M Karkar, L.D Leary ,O.V Lie , M. Girouard ET AL , Electromyography-based seizure detector: preliminary results comparing a generalized tonic-clonic seizure detection algorithm to video-EEG recordings , Epilepsia ,56(9), (2015), pp. 1432-1437.

[19] J. Arends , R.D. Thiss ,T. Gutter , C. Ungureanu , P. Cluitmans , J. Van Dijk et al, Multimodal nocturnal seizure detection in a residential care setting, Neurology 91(21), (2018), pp. e2010-e2019.

[20] G. Regalia , F. Onorati, M. LAi , C. CABORni, R.W.PiCARD ET AL, Multimodal wrist-worn devices for seizure detection and advancing research: focus on the Empatica wristbands. , Epilepsy Res 153, (2019), pp. 79-82.

[21] M.Z. Poh, T. Loddenkemper, C, Reinsberger, NC. Swenson, S. Goyal, M.C. Sabtala et Al, Convulsive seizure detection using a wrist-worn electrodermal activity and accelerometry biosensor, Epilepsia, 53(5), (2012), pp. 93-97

[22] J. LiU, Z. LiU, H. ZhANG, ET AL, Multi-sensor information fusion for IoT in automated guided vehicle in smart city, Soft Comput, (2021), 25(2):1-13

[23] S.Sguazza, Sensor Data Synchronization in a IoT Environment for Infants Motricity Measurement, IoT Technologies for HealthCare, (2019), Vol 3

[24] P.Sethi And S.R. SARAngi, Internet of Things: Architectures, Protocols, and Applications, Soft Comput, (2017), 9324035

[25] M. Garbarino, M. Lai, D. Bender, R.W. Picard, Et Al, Empatica E3 - A wearable wireless multi-sensor device for real-time computerized biofeedback and data acquisition, ICST Transforming healthcare through innovations in mobile and wireless technologies (2014), 39-42

[26] W. Xu, C. Yan, W. JiA, Analyzing and Enhancing the Security of Ultrasonic Sensors for Autonomous Vehicles, IEEE Internet of Things Journal, (2018), 5(6), pp. 2015-2029.

[27] J. Bai, S. Li, L. HuAng And H. Chen, Robust Detection and Tracking Method for Moving Object Based on Radar and Camera Data Fusion, IEEE Sensors Journal, 21(9), (2021),pp. 10761-10774

[28] A.M. Rahmani, T.N Gia, B. Negash, A. Anzanpour, I. Azimi, M. Jiang, P. Liljeberg, Exploiting smart e-Health gateways 
at the edge of health-care Internet-of-Things: A fog computing approach, Future Generation Computer Systems, 78(2), (2018) pp. 641-658

[29] M. Maheepala ,M.A. Joordens, A.Z. KouZani, Low Power Processors and Image Sensors for Vision-Based IoT Devices: A Review, IEEE SENSORS JOURNAL (2021), 21(2), pp. 1172-1186

[30] M. Bansal and Priya , Application Layer Protocols for Internet of Healthcare Things (IoHT) , 2020 Fourth International Conference on Inventive Systems and Control (ICISC), (2020), pp. 369-376.

[31] G. Marques, R. Pitarma, M. Garcia, N. Pombo,Internet of Things Architectures, Technologies, Applications, Challenges, and Future Directions for Enhanced Living Environments and Healthcare Systems: A Review, Electronics, 8(10), (2019), pp 1081

[32] M. Ijaz; G. Li.; H. Wang; A.M. El-Sherbeeny; Y.M. Awelisah., L. Lin, A. KoubaA, A. Noor Intelligent Fog-Enabled Smart Healthcare System for Wearable Physiological Parameter Detection, Electronics, 9(12), (2015), pp 2015

[33] R. Dautov, S. Distefano, and R. Buyya Hierarchical data fusion for Smart Healthcare, J Big Data, 6, (2019)

Edited by: Pradeep Kumar Singh et al.

Received: May 31, 2021

Accepted: Oct 18, 2021 